\title{
A measurement system for the long-term diagnostics of the thermal and technical properties of wooden houses
}

\author{
Jakub Svatos $^{1}$, Jan Holub ${ }^{1}$, Tomas Pospisil ${ }^{1}$ \\ ${ }^{1}$ Czech Technical University, Faculty of Electrical Engineering, Dept. of Measurement, Technicka 2, 16627 Prague 6, Czechia
}

\begin{abstract}
To reduce construction costs and to increase energy savings and indoor environmental quality in a wooden house, an energy consumption measuring system for the diagnostics of the thermal and technical properties of building envelopes has been developed. At present, the vast majority of calculations for the thermal and technical characteristics of buildings are based on simple mathematical models of building envelope behavior, and these are derived primarily from the thermal transmission of individual materials. However, thermal transmission is measured in a stable laboratory environment. Therefore, the measured values may not match the real behavior of materials in continually changing climatic conditions. The energy consumption measuring system was installed in seven wooden houses in different climatic areas to diagnose the thermal and technical properties of a building envelope. The power consumption, temperature, and humidity of the air as well as of the wood; $\mathrm{CO}_{2}$ concentration; the temperature of the individual layers of the envelope sandwich structure; and wind speed and direction were measured. Thanks to the sets of real measurements of the measured thermal and technical behavior, the actual dependence of the indoor environment quality on the outdoor climatic conditions can be ascertained. The presented system can reveal imperfections in the heat capacity and hygroscopicity of materials used for the construction of a wooden house. Based on this measurement, the materials can be revised to save construction costs and energy; to increase the indoor environment quality; and to reduce the impact on the environment by decreasing the amount of used materials.
\end{abstract}

Section: RESEARCH PAPER

Keywords: measuring system; thermal propagation; wooden house measurement; indoor environment quality

Citation: Jakub Svatos, Jan Holub, Tomas Pospisil, A measurement system for the long-term diagnostics of the thermal and technical properties of wooden houses, Acta IMEKO, vol. 9, no. 3, article 2, September 2020, identifier: IMEKO-ACTA-09 (2020)-03-02

Editor: Jan Saliga, Technical University of Košice, Slovakia

Received December 12, 2019; In final form March 5, 2020; Published September 2020

Copyright: This is an open-access article distributed under the terms of the Creative Commons Attribution 3.0 License, which permits unrestricted use, distribution, and reproduction in any medium, provided the original author and source are credited.

Corresponding author: Jakub Svatos, e-mail: svatoja1@fel.cvut.cz

\section{INTRODUCTION}

This article presents a building energy consumption monitoring system for the determination of a heat transfer through the individual parts of a wooden house envelope for the diagnostics of the thermal and technical properties of buildings.

The increasing demand for energy-efficient buildings specifically wooden houses - during the past decade has caused a higher standard of the thermal insulation of building envelopes. Buildings are responsible for $40 \%$ of the energy consumption in the EU [1]. At present, there is no binding uniform methodology for the diagnostics of the wooden house sandwich structure system. These characteristics have been tested in the accredited laboratories in laboratory conditions, where real climatic changes are only simulated. Due to the fact that only the surface temperature of the tested object (the envelope sandwich structure) is measured and the other quantities such us heat flux density or heat transfer coefficient are implicitly calculated, these results do not correspond to actual conditions. Besides, the subjective and objective causes of the use of wooden houses are omitted. In newly built or reconstructed residential buildings there is very often excessive over-dimensioned thickness of insulation systems $(200 \mathrm{~mm}, 250 \mathrm{~mm}$, etc.). The energy saving in such an over-dimensioned building is already negligible, and the hygienic comfort of a user is clearly deteriorating [2]-[4].

Many studies have dealt with calculation procedures or have presented software or algorithms to estimate heat losses based on mathematical models. When different calculations for energy losses based on various mathematical models [5]-[10] are compared, it is evident the reliability of these simplified models and standards is low.

The technical solution proposed herein could eliminate the shortcomings of the currently available solutions in the area of the energy consumption of wooden houses. To introduce a binding uniform methodology based on the diagnostics of the thermal and technical properties of wooden houses, a set of 
different quantities monitoring indoor and outdoor environment must be analysed. The system should also include a set of sensors for monitoring the thermal propagation through the individual layers of the building envelope. Moreover, sensors monitoring the temperature and humidity of the wood inside the building's structure are desirable [11]-[14].

With the increasing proportion of wooden houses among the total number of buildings constructed (e.g. in Scandinavia, Japan, and the US, more than $70 \%$ of newly constructed buildings are wooden houses), there is a need to monitor the relative humidity and the humidity of the wood (to prevent the increase of humidity); $\mathrm{CO}_{2}$ concentration; and temperature in order to improve indoor environment comfort [8].

This article introduces a complete measuring system that can monitor and measure indoor environment quality and diagnose the thermal and technical properties of building envelopes related to outdoor weather conditions. The proposed system also includes a developed application for collecting and processing all the measured data. If sufficient data is collected from wooden houses built in different relevant climatic areas, the binding uniform methodology can reduce construction costs, increase energy savings, and improve the indoor environment quality of wooden houses.

The article is organised as follows. In Section II, the energy consumption measuring system for diagnostics and the developed software are introduced and described. In Section III, the example of measured real data over a year is presented. A conclusion and future work directions are presented in Section IV.

\section{METHODS}

In cooperation with the construction company RD Rymarov s. r. o., seven different one- and two-storey residential wooden houses were selected for diagnosis for more than three years. Moreover, a modified guarded hot box was installed to compare and verify the obtained results. The hot box model can also carry out the full-scale testing of the entire building systems well and is therefore valid for comparison with real conditions. This model includes the same measurement system setup as a one-

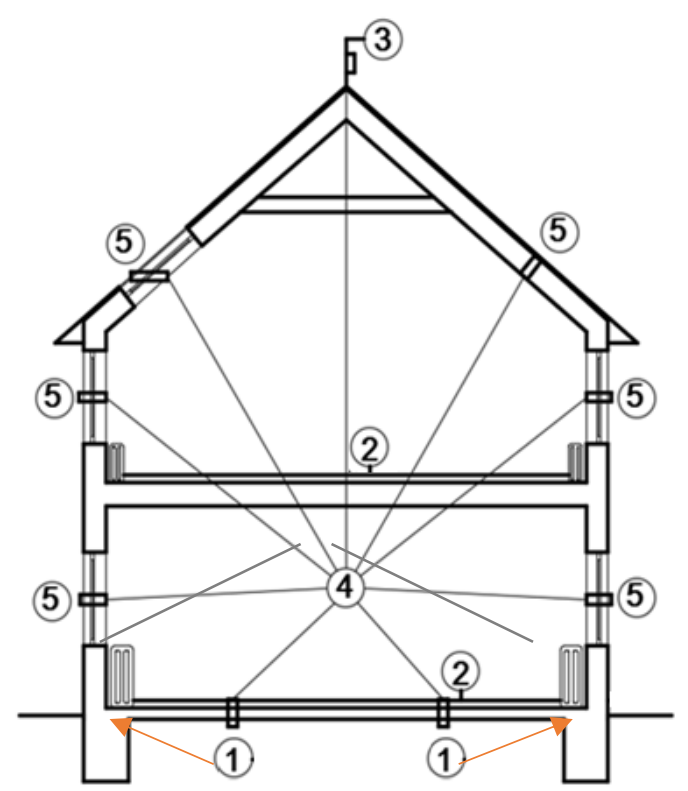

Figure 1. The positioning of the measuring sensors. storey wooden house. The one-storey wooden house has been measured, processed, and presented by Czech University of Life Science in Prague [15].

All the selected buildings are located in the Czech Republic in four different climatic areas - in order to provide a complex measurement for the proposed methodology. The analysis was performed over a three-year period in order to collect sufficient data across the seasons.

The proposed measuring system involves monitoring the weather (specifically the wind speed and its direction); the outside temperature and air humidity; the power consumption of the whole building and of the heating units only; the indoor temperature and relative air humidity; $\mathrm{CO}_{2}$ concentration; wood temperature; and moisture. To monitor the thermal propagation through the sandwich structure of the envelope of the wooden house, the temperature in at least three different layers of the structure was also required to be monitored. Moreover, the system also includes all necessary communication units, collecting data in a monitored object. The data from all the monitored buildings must be measured in real time and wirelessly sent to a remote server. The data is processed and stored for further diagnostics. Moreover, the measured data is supplemented by the local weather forecast.

The proposed system has been designed in order to fulfil all the above-mentioned criteria, with the aim of measuring the outdoor and indoor environment as well as the thermal propagation through the envelope and the wall sandwich structure of the residential wooden house. Since the system must monitor the object for at least three years and since most of the sensors are placed into a structural system where they cannot be reached, it was decided not to use the wireless connection of individual sensors to the central unit, since the loss of the data (due to e.g. loss of power or the disturbance of a wireless transmission) could seriously affect the experiment.

\subsection{Measured system}

An example of a typical set of sensors and its positioning around a two-floor wooden house is shown in Figure 1.

Each monitored object has its own central unit (4 in Figure 1), where all the sensors are connected via the RS-485 (a standard for use in serial communications systems) or the ethernet. This central unit secures the wireless communication with the remote server, where all the measured data is stored.

The outdoor environment is measured by the temperature and relative humidity of the air sensor and ethernet anemometer, which measures wind speed and its direction, placed $1 \mathrm{~m}$ above the rooftop of the monitored wooden house ( 3 in Figure 1).

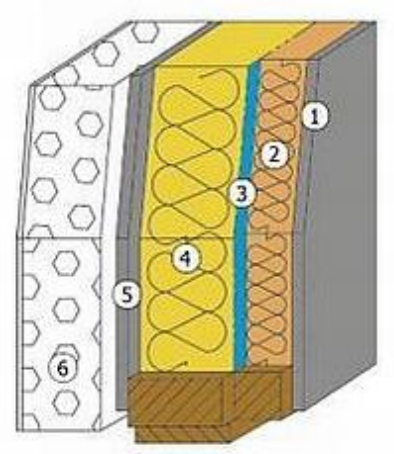

Figure 2. The scheme of the standard wall of a wooden house [16]. 


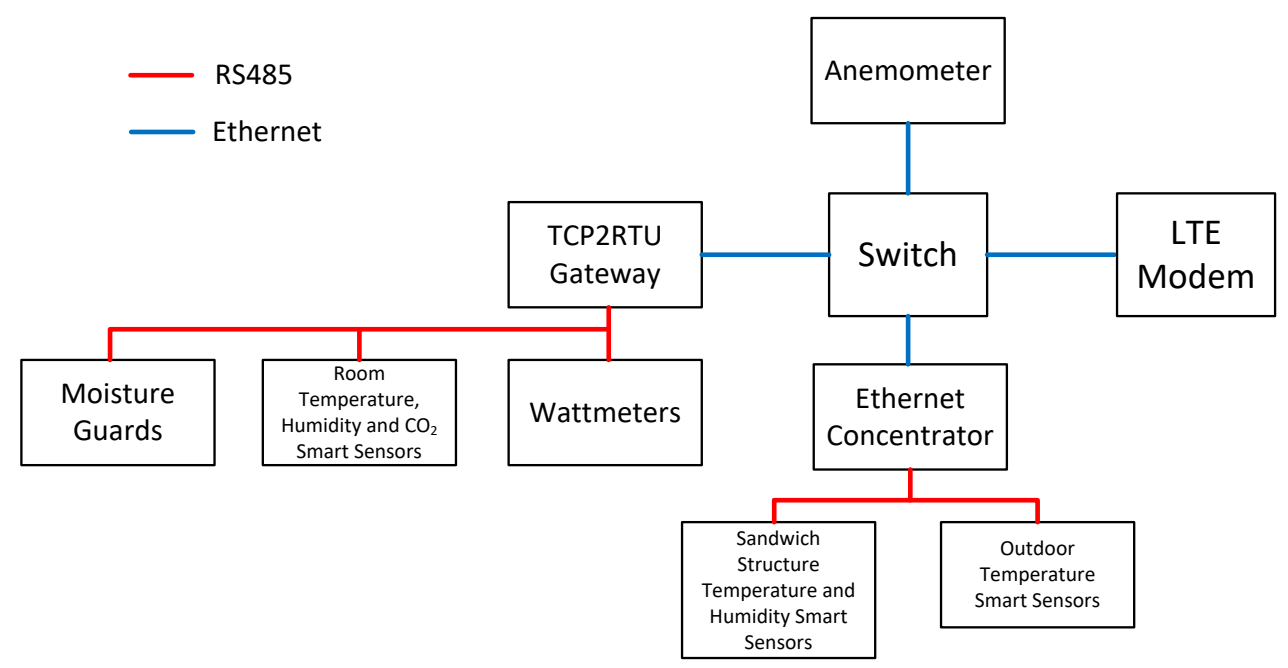

Figure 3. A block diagram of the measurement system.

According to the requirement to measure the outdoor environment in such a way that the proposal of this new methodology of wooden house diagnostics is possible, a set of the temperature and relative humidity of the air sensors is used. The sensors are placed at the outer part of the envelope sandwich structure, specifically in the thermo-facade (5 in Figure 2), at each cardinal direction, and on every floor - at a distance of about 1.5 $\mathrm{m}$ above the base level of each floor, including the roof (5 in Figure 1), as shown in Figure 1.

To monitor the thermal propagation through the envelope and the wall sandwich structure of the wooden house, two additional layers of the wall are sensed.

Each wooden house sandwich envelope consists of a fermacell ${ }^{\circledR}$ gypsum fibreboard (1), a wooden frame filled with heat insulation (2), a vapour barrier (3), a wooden frame filled with heat insulation (4), a fermacell ${ }^{\circledR}$ gypsum fibreboard, (5) and a thermo-facade (6), as shown in Figure 2.

The temperature and humidity of the air inside the envelope in the wooden frame filled with heat insulation (4 in Figure 2) and in the fermacell ${ }^{\circledR}$ gypsum fibreboard (1 in Figure 2$)$ are also measured. In this way, the thermal propagation/heat flux through the sandwich structure is monitored on each floor of the building, including the roof and on all sides of the object. To ensure the sensors do not come into contact with the heat insulation and to measure the real temperature and the humidity of the air, all sensors placed inside the sandwich structure are placed in a small cage. The accuracy class of all the air temperature sensors used is $\pm(0.3+0.005|v|)$, where $v$ is the measured temperature in ${ }^{\circ} \mathrm{C}$.

Besides the measurement of the temperature and humidity of the air in the sandwich structure in all cardinal directions, the temperature and humidity of the wooden structure are also measured. The four moisture guard intelligent sensors are placed in an area where the structural system of the wooden house meets the baseplate ( 1 in Figure 1). Further details about the moisture guard system can be found in [14], [17].

To collect the data from the indoor environment and the temperature and humidity of the air, $\mathrm{CO}_{2}$ concentration smart sensors ( 2 in Figure 1) were installed in each room of the wooden house. $\mathrm{CO}_{2}$ concentration sensors help monitor the movement of residents in the house and can reveal the quality of the air inside the individual rooms. The $\mathrm{CO}_{2}$, a result of the metabolic process in living organisms, and its concentration within a room is often used to indicate whether adequate fresh air is being supplied to space and is used as a significant indicator of air quality. Moderate to high levels of carbon dioxide can cause headaches; difficulty in breathing, increased heart rate and fatigue; and even higher concentrations can produce nausea, dizziness, and vomiting. The $\mathrm{CO}_{2}$ level and the potential health problems associated with high $\mathrm{CO}_{2}$ levels are shown in Table 1 [18].

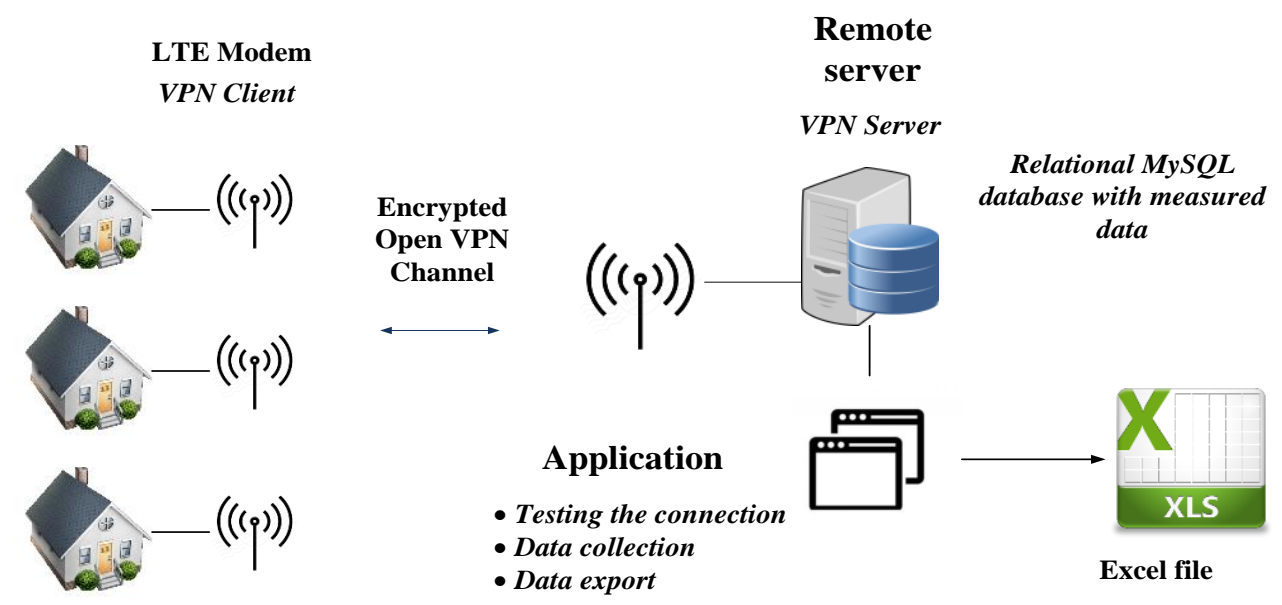

Figure 4. The data collection block diagram. 
Table 1. $\mathrm{CO}_{2}$ concentration and its effects.

\begin{tabular}{cl}
\hline $\begin{array}{c}\mathrm{CO}_{2} \text { concentration level } \\
(\text { ppm) }\end{array}$ & \multicolumn{1}{c}{ Effects } \\
\hline $250-350$ & The normal outdoor level \\
$350-1000$ & Occupied indoor spaces with air exchange \\
$1000-2000$ & Complaints of drowsiness and poor air \\
$2000-5000$ & $\begin{array}{l}\text { Poor concentration, loss of attention, } \\
\text { increased heart rate }\end{array}$ \\
$>5000$ & An unusual air condition - toxicity could occur
\end{tabular}

In general, ventilation rates should keep $\mathrm{CO}_{2}$ concentrations below $1000 \mathrm{ppm}$ to ensure that the indoor air quality is acceptable for most inhabitants. The accuracy of the sensor used in this experiment is $\pm(50 \mathrm{ppm}+2 \%$ from reading $)$ and the measuring range is from 0 to $2000 \mathrm{ppm}$, since it is not expected to overreach the typical environmental limits of a house.

Intelligent power meters for energy consumption monitoring were also present. One power meter is used solely for the measurement of individual rooms heating and the other measures the overall energy consumption of the residential wooden house.

All the intelligent sensors for the outdoor measurement and the measurement of the sandwich structure use the same ethernet concentrator in order to collect the data at the central unit. Since the RS-485 standard allows a maximum number of 32 devices to be connected (which is less than the projected number of sensors), the ethernet concentrators are used to extend the maximum number of devices/intelligent sensors. In this way, the total number of connected sensors can exceed 100 .

The moisture guards, power meters, and the temperature and air humidity sensors for monitoring the indoor environment quality use a RS-485 bus and TCP2RTU gateway (TCP Transmission Control Protocol, RTU - Remote Terminal Unit) for connection to the central unit. The TCP2RTU is a transparent bidirectional converter of the Modbus TCP protocol (Modbus - a data communications protocol), which runs by means of the ethernet. The moisture guard is connected via the RS-485 to the TCP2RTU gateway. The ethernet concentrator and the TCP2RTU gateway are then connected to the industrial ethernet switch, which is connected to the communication unit and sends the measured data wirelessly to the remote server using

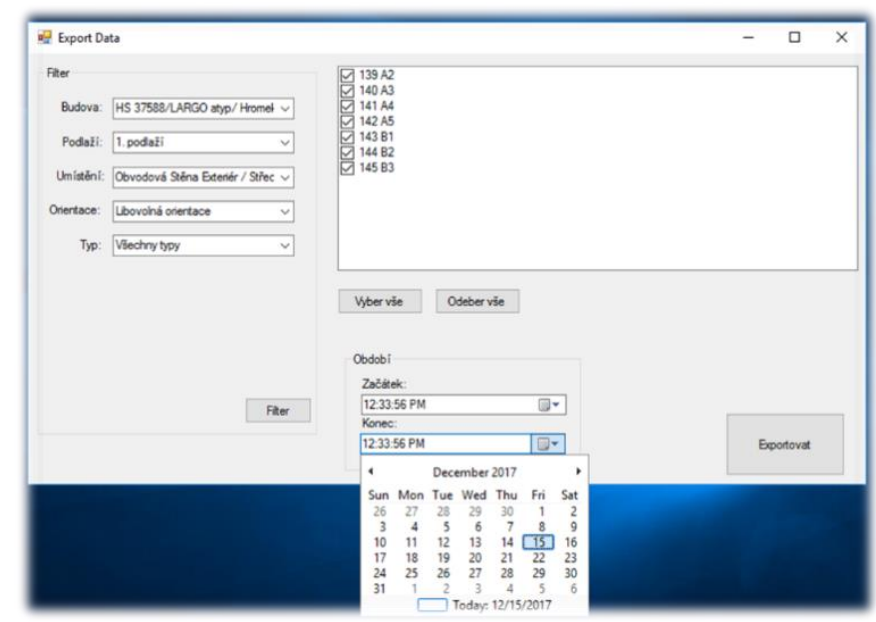

Figure 5. The application used for exporting the measured data.

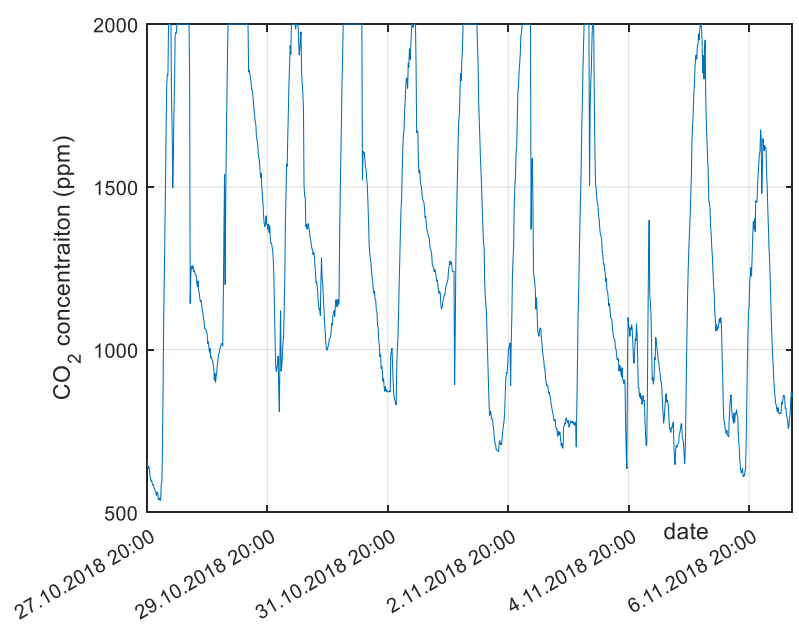

Figure 6. $\mathrm{CO}_{2}$ concentration in the master bedroom.

the LTE standard (LTE - Long-Term Evolution, a telephone and mobile broadband standard). A block diagram of the complete system is shown in Figure 3. The system is also designed in such a way that a power supply of $+12 \mathrm{~V}$ is used.

As explained above, communication within one monitored object is carried out by using the RS-485 Fieldbus, mainly due to its high resistance to disturbance. To ensure compatibility between individual sensors, data transfer from sensors or measuring transducers is done by using the Modbus RTU protocol. Due to the requirement to send measured data in real time to a remote server, the TCP2RTU gateway is added to the measuring chain. The Modbus TCP uses a standard ethernet cable as a physical layer and allows remote access to all sensors or measuring transducers using the internet. All TCP2RTU gateways are connected to an industrial switch, which is connected to an LTE modem. The LTE modem sends data across a mobile $3 \mathrm{G}$ network to a remote server.

\subsection{Communication and software}

The measured data acquisition is performed using a clientserver network architecture. The principle of the data acquisition from the monitored residential wooden houses and the laboratory model is shown in Figure 4. As part of the proposed system, a custom software package has been developed. The key element of the package is a server application that is used for data collection and storage. The application periodically connects clients (the monitored object) and the respective intelligent sensors, and it provides continuous data collection. The application has been designed as a multi-threaded application, in which each thread handles one monitored object.

The acquired data is stored in a relational SQL database by the server application (SQL - Structured Query Language, a language for relational database systems). The database is located on the server and stores aggregated information about the monitored objects, installed sensors (their positions, measured quantities, communication addresses, etc.), and measured data from all the sensors. The data contains measured values and includes the time stamp and a link to a particular sensor.

The overall number of the sensors used depends on the number of rooms and floors. The average count of sensors per single monitored object is 63 . The server application acquires the data periodically with a sample frequency of $f_{\mathrm{s}}=0.0011 \mathrm{~Hz}$, i.e. four samples per hour. In this way, more than 80000 measured samples are collected per day (for all monitored objects in the study). 
Another application of the developed software package is used for ease of data export from the database (Figure 5).

In the form, a user can select a specific monitored object, a time interval, and a specific sensor(s) that can be filtered by different criteria - the building, the floor of the building, the position of the sensor, cardinal direction orientation, and the type of sensor. The selected data is then exported from the database to a structured MS Excel spreadsheet or CSV file.

The last two applications are used to set up the communication parameters of the intelligent sensors before their installation into the monitored objects and to control communication during the installation of all sensors into the monitored objects.

\section{RESULTS}

The results presented here were collected from one monitored fully inhabited residential wooden house located in the highland in the eastern part of the Czech Republic in elevation of $208 \mathrm{~m}$. The data was collected between 2018-2019.

From all the measured data mentioned in Section II, the $\mathrm{CO}_{2}$ concentration, the change in the humidity of the wood, the temperature in the envelope sandwich structure in different parts of the object, and stove heating monitoring were selected as examples to demonstrate the relevance of data from real-life scenarios.

\section{1. $\mathrm{CO}_{2}$ concentration}

A resident's habits and movement around the house can be revealed by monitoring $\mathrm{CO}_{2}$ concentration. The data clearly shows the presence of the residents and the durations of the ventilation or when the residents leave the object. Therefore, it gives an understanding the connection between the movement of the residents around the house and the indoor temperature and ventilation.

The first example of the $\mathrm{CO}_{2}$ concentration monitoring is that which was done in the master bedroom over 14 days, shown in Figure 5.

The presented results show the problem of modern wooden houses. If there is no frequent ventilation, the $\mathrm{CO}_{2}$ concentration limits are very often exceeded; therefore, potential health problems can occur, as shown in Table 1 . This problem can be solved by increasing the awareness of home users of the need to ventilate their homes often or by means of an automatic ventilation system. Figure 7 presents the measured data in the

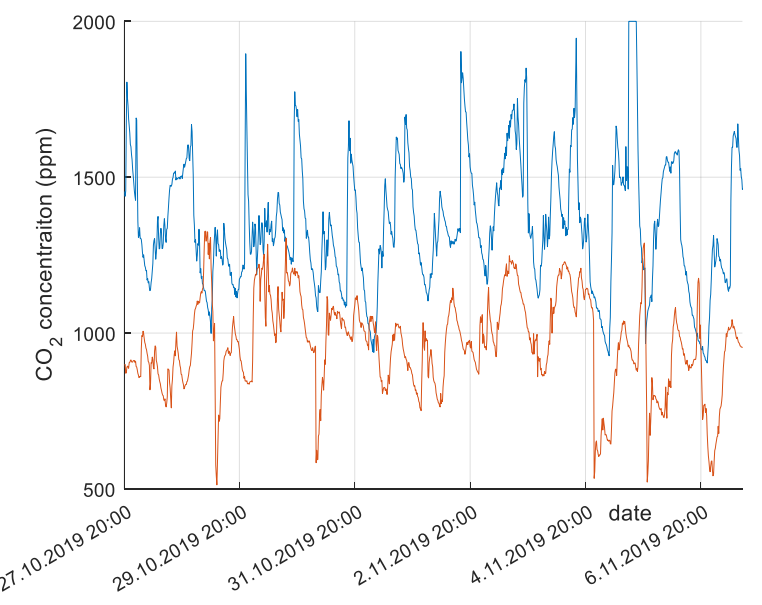

Figure 8. $\mathrm{CO}_{2}$ concentration in master bedroom (blue) and kid bedroom (red).

same 14-day period one year later, when an automatic ventilation system was already in use. The graph shows the $\mathrm{CO}_{2}$ concentration values in the master bedroom (the blue curve) occupied by two persons with proper ventilation and the child's bedroom (the red curve), occupied by only one person.

The presented data confirms the possibility of using $\mathrm{CO}_{2}$ concentration sensors to monitor the activity of the residents around the house and even estimate the number of people there.

\subsection{The humidity of the wood}

The humidity of the wood measured directly by moisture guards can help to better determine the quantities, such as heat flux density or the heat transfer coefficient.

The measurement of the moisture of the wooden house sandwich structure started shortly after finishing the construction and move of inhabitants into the house. The moisture guards are located in a place in which the structural system of the wooden house meets the baseplate, and all cardinal directions of the monitored object are located there.

Figure 8 shows the change of the moisture over the ninemonth period in all cardinal directions. The result revealed the change of the moisture right after finishing the construction, where the change is approximately $3 \%$.

Figure 9 presents the trend of the wood humidity changes over the second year of the monitoring, when the moisture had already stabilised.

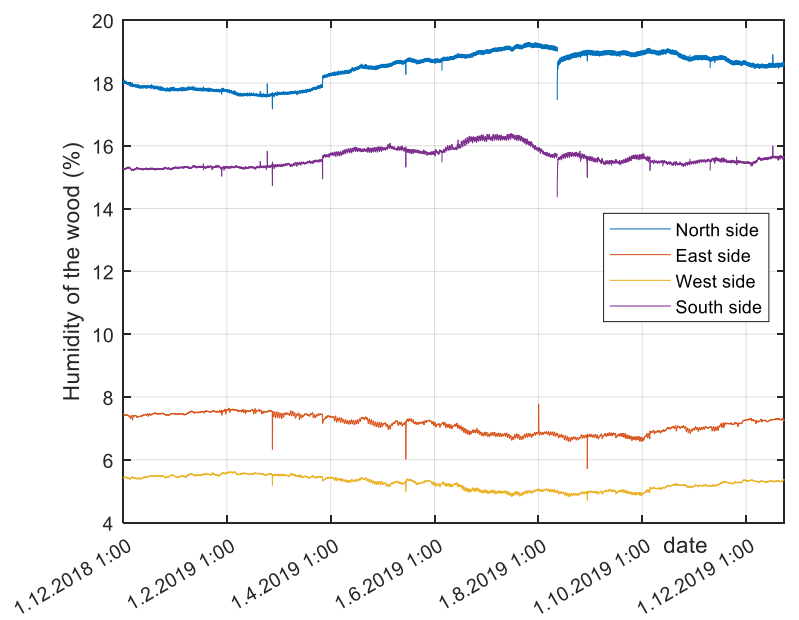

Figure 9. Changes in the humidity of the wood.

Figure 7. Changes in the moisture of the wood. 
The moisture guards' data are also valuable for the object's inhabitants for early detection of moisture in the building's structure.

\subsection{Temperature in the building envelope sandwich structure}

The building envelope sandwich structure is permanently in direct contact with the outdoor atmosphere and the indoor conditions on the other side. To monitor the temperature in this part of the structure gives insight into complete building systems as they are used in real conditions.

Figure 10 to Figure 15 show the changes in temperature through the individual layers (see Figure 2) of the building envelope sandwich structure in different locations of the house. The measured values can be used to calculate the total energy leaked through the monitored object compared with calculated values according to the boundary conditions. Figure 10 and Figure 11 present the temperatures in the individual layers of the envelope in two cardinal directions - the north- and southoriented walls on the first floor.

The differences between the south and north sides during the four seasons are immediately noticeable, especially in the middle of the sandwich structure (a wooden frame filled with heat

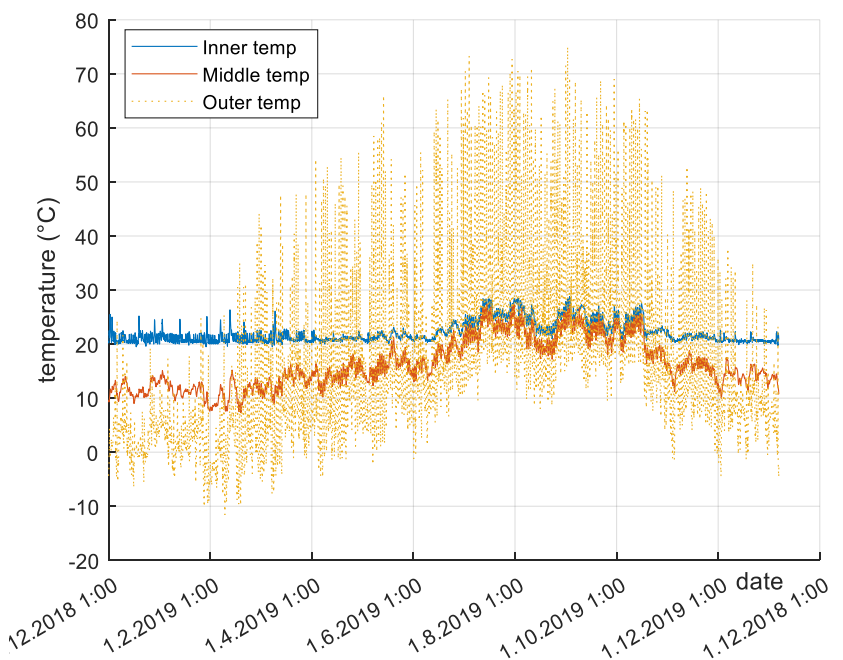

Figure 10. The temperature in the individual layers of the building envelope sandwich structure that are oriented north.

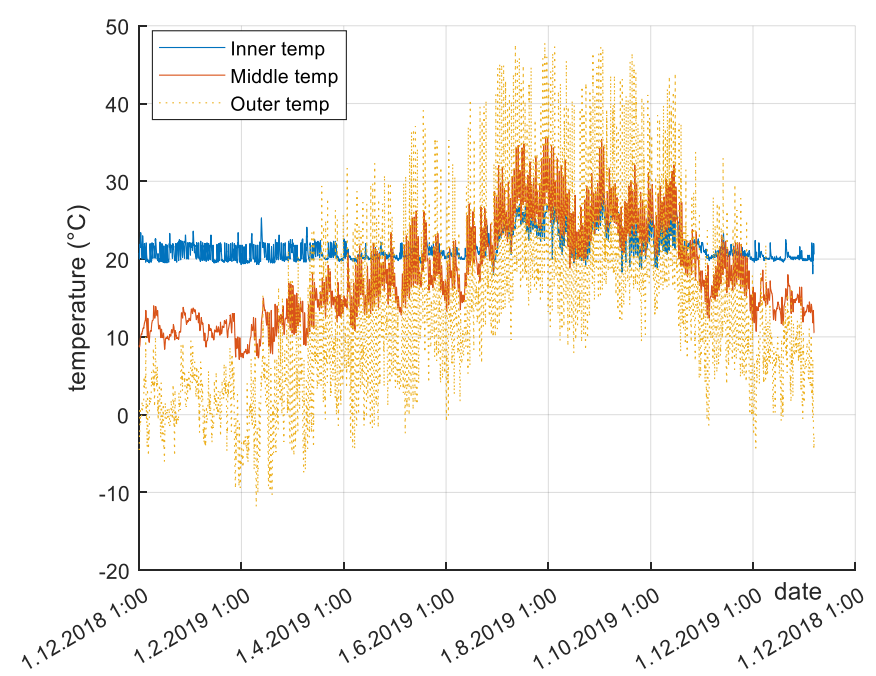

Figure 11. The temperature in the individual layers of the building envelope sandwich structure that are oriented south. insulation). The detail of the north side over a one-month period, where the change of the temperature in the individual layers every day is clearly visible, as presented in Figure 12.

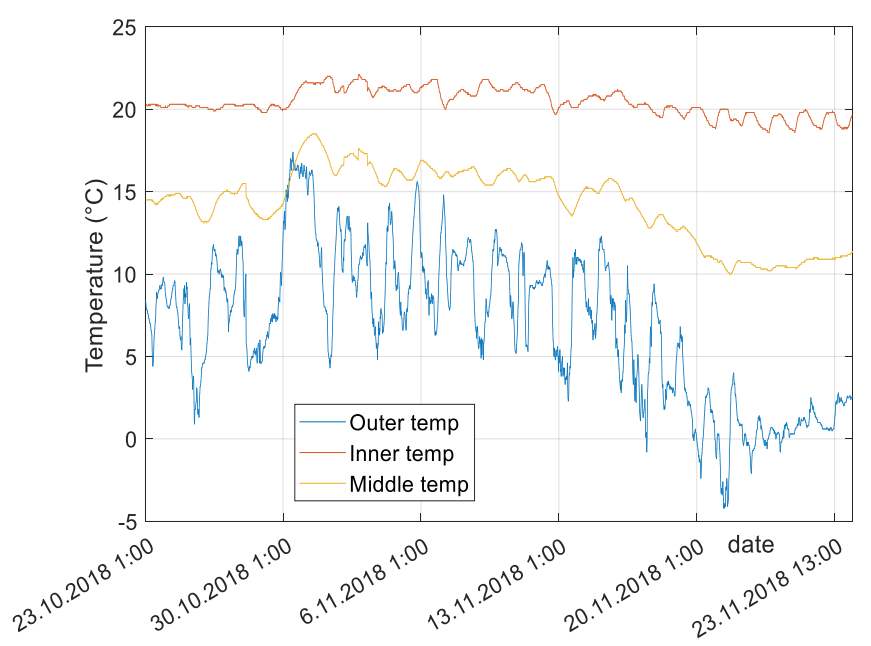

Figure 12. The temperature in the individual layers of the building envelope sandwich structure that are oriented south.

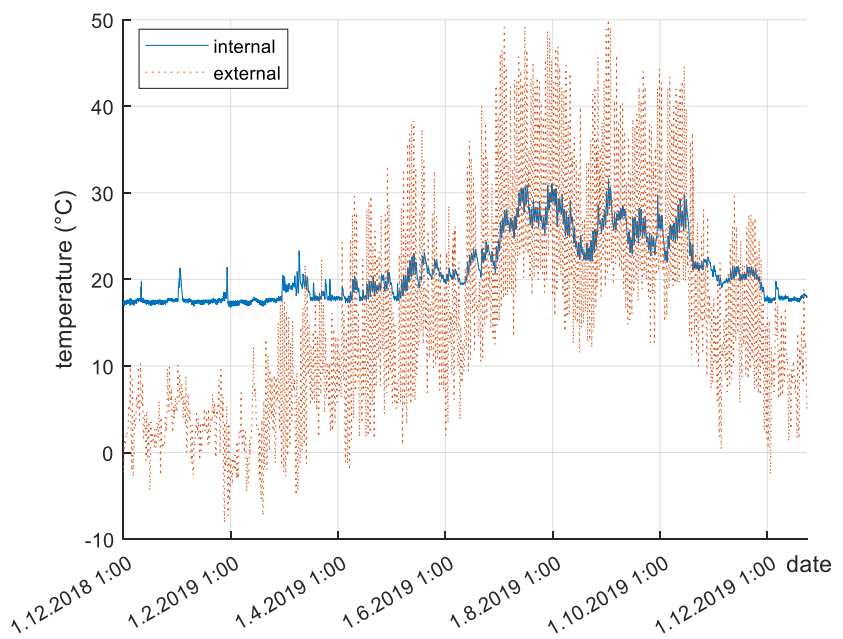

Figure 13. The temperature in the individual layers of the roof sandwich structure that are oriented north.

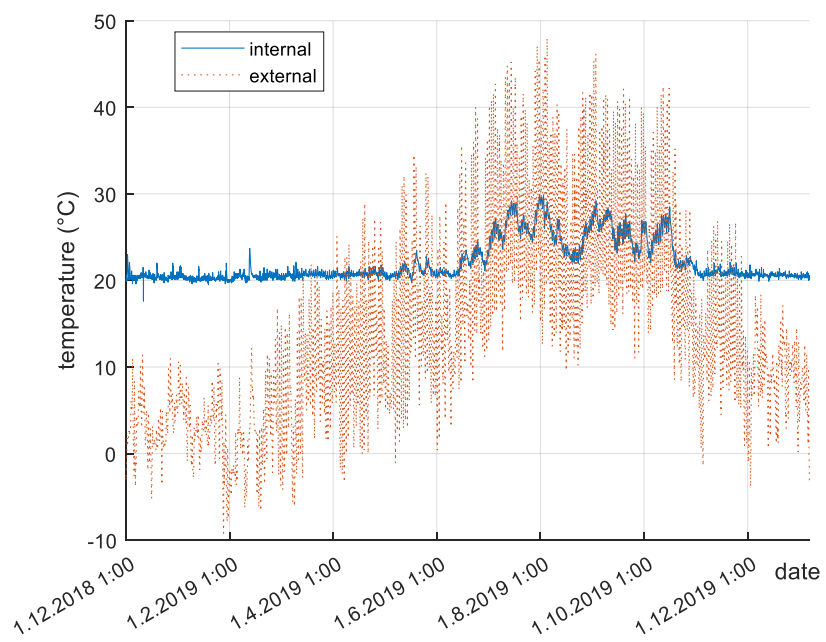

Figure 14. The temperature in the individual layers of the roof sandwich structure that are oriented south. 


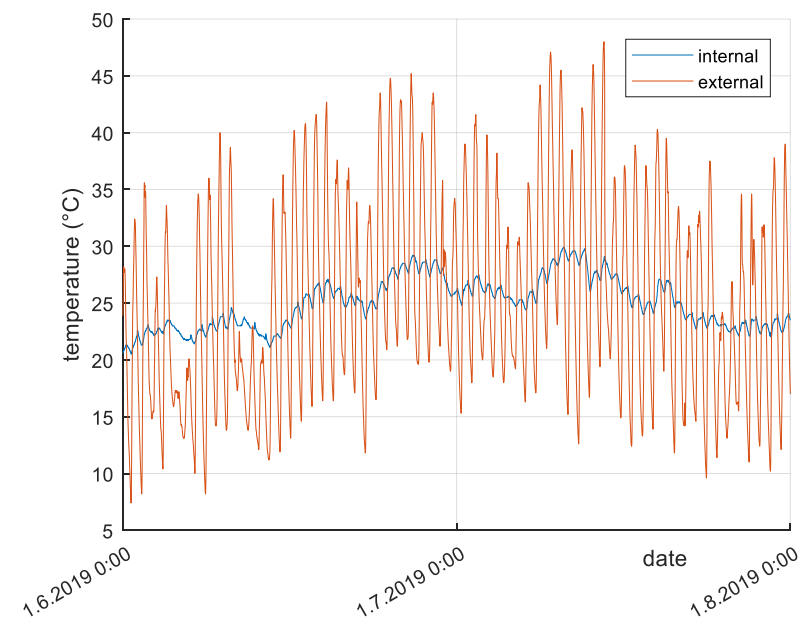

Figure 15. The temperature in the individual layers of the roof sandwich structure that are oriented south.

The changes in the temperature in the two layers of the roof over the same period are presented in Figure 13 and Figure 14. Figure 15 shows the detail over a two-week period, similar to Figure 12.

The data presented in Figure 16 illustrates the changes in the temperature near the chimney. Usage of the hearth provides complex knowledge about the heating in the whole building.

Using this data, the thermal propagation/heat flux, the thermal conductivity, and the energy losses of the object can be calculated [3], [19]. Based on these measurements, a binding uniform methodology for the diagnostics of a wooden house sandwich structure system could be proposed and unite the methodology in order to save on construction costs and increase the indoor environment quality for the inhabitants. In the future, the system can be used for commercial use in order to monitor and diagnose any wooden structure or for it to be extended to be compatible with IoT [20]. Based on the measurement, the ventilation, heating, or cooling systems can be controlled. Furthermore, the proposed system can also monitor the humidity of the wooden structure, and it will be able to detect moisture in a building structure early.

\section{CONCLUSIONS}

In the article, an energy consumption measuring system for diagnostics has been introduced. The complete solution consists of the design of a universal measurement system for monitoring the thermal and technical properties of individual parts of the wooden house building envelope; the energy consumption for heating or cooling; and the total energy consumption of the building, depending on the real climatic conditions to which the building is exposed.

The energy consumption measuring system for diagnostics has an innovative contribution due to the well-designed measurement methodology and its evaluation. The measured results correspond to actual climatic changes in combination with indoor environmental conditions. Based on the analysis, the individual components of a wooden house building envelope can be revised to save the construction costs, save energy, reduce the impact on the environment by decreasing the amount of used materials, and increase the indoor environment quality of the wooden houses. This approach can provide the real thermal

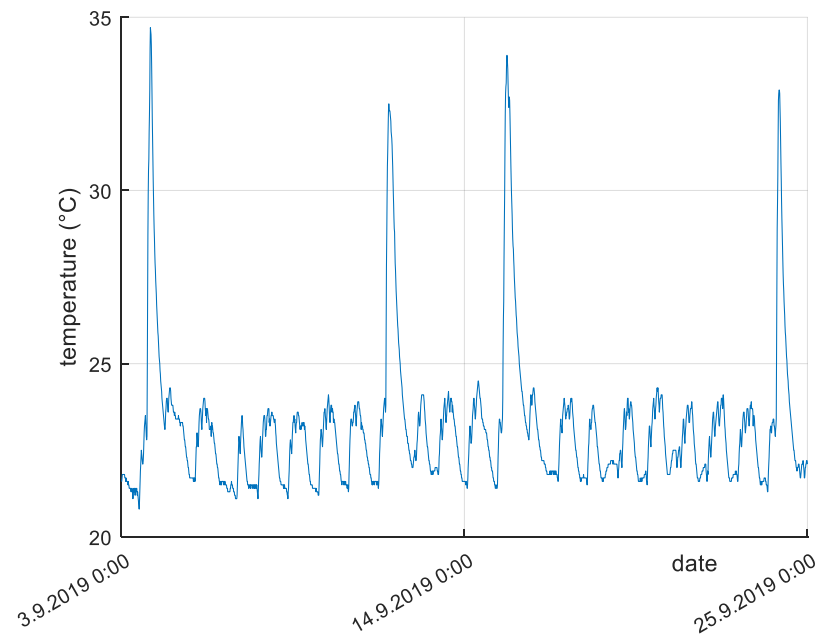

Figure 16. The temperature near the chimney.

conditions and help in revealing imperfections in the heat capacity and hygroscopicity of the materials used.

The results of this project demonstrate the method for the full-scale energy performance of an object's walls, ceiling, or roof system in the dynamic conditions of the real environment.

\section{ACKNOWLEDGEMENT}

This research was supported by the TH02020792 'Development of the methodology of estimation of interior heat parameters by the wood residential constructions, reduction of the energy intensity and environmental factors related of the reducing of the greenhouses gases' grant provided by the Technology Agency of the Czech Republic.

\section{REFERENCES}

[1] K. Trgala, M. Pavelek, R. Wimmer, Energy performance of five different building envelope structures using a modified Guarded Hot Box apparatus - comparative analysis, Energy and Buildings 195 (2019) pp. 116-125.

DOI: https://doi.org/10.1016/i.enbuild.2019.04.036

[2] J. Šála, L. Keim, Z. Svoboda, Z. Tywoniak, Thermal Protection of Buildings, IC CKAIT s.r.o., 2008, ISBN: 978-80-87093-30-6.

[3] M. Kloiber, M. Drdácký, Diagnostics of Wooden Constructions, IC CKAIT s.r.o., 2016, ISBN: 978-80-87438-64-0.

[4] ČSN 73 0540-3 Thermal Protection of Buildings, Czech Standards Institute, 2005.

[5] R. D. L. Vollaro, C. Guattari, L. Evangelisti, G. Battista, E. Carnielo, P. Gori, Building energy performance analysis: a case study, Energy and Buildings 87 (2015) pp. 87-94.

DOI: https://doi.org/10.1016/j.enbuild.2014.10.080

[6] N. Aste, A. Angelotti, M. Buzzetti, The influence of the external walls thermal inertia on the energy performance of well insulated buildings, Energy and Buildings 41(11) (2009) pp. 1181-1187. DOI: https://doi.org/10.1016/j.enbuild.2009.06.005

[7] E. Kossecka, J. Kosny, Influence of insulation configuration on heating and cooling loads in a continuously used building, Energy Buildings 34(4) (2002) pp. 321-331.

DOI: https://doi.org/10.1016/S0378-7788(01)00121-9

[8] P. Bacher, H. Madsen, Identifying suitable models for the heat dynamics of buildings, Energy and Buildings 43(7) (2011) pp. 1511-1522.

DOI: https://doi.org/10.1016/j.enbuild.2011.02.005

[9] E. Enache-Pommer, R. Mayer G. Parsons, Energy efficiency of building walls: thermal modelling, experimental testing, long term 
evaluation and correlation of building wall systems, ASHRAE Trans. 119 (2013).

[10] Y. Feng, Thermal design standards for energy efficiency of residential buildings in hot summer/cold winter zones, Energy and Buildings 36(12) (2004) pp. 1309-1312. DOI: https://doi.org/10.1016/i.enbuild.2003.08.003

[11] J. Blazek, M. Mucka, K. Trgala, Cost and thermal-technical optimization of wooden construction in the passive standard, Wood Research 61 (2016) pp. 663-672.

[12] United States Department of Agriculture, Wood Handbook Wood as an Engineering Material, U.S. Forest Service, General Technical Report FPLGTR190, 2010.

[13] D. Onysko, C. Schumacher, P. Garrahan, Field Measurements of Moisture in Building Materials and Assemblies: Pitfalls and Error Assessment, DMO Associates, Building Science Corporation, FPInnovations, Forintek Division, 2008.

[14] J. Včelák, A. Vodička, M. Maška, J. Mrňa, Smart building monitoring from structure to indoor environment, Proc. of the Smart City Symposium Prague (SCSP), Prague, 25-26 May 2017, 5 pp. DOI: https://doi.org/10.1109/SCSP.2017.7973859

[15] C. Buratti, E. Belloni, L. Lunghi, M. Barbanera, Thermal conductivity measurements by means of a new 'Small Hot-Box' apparatus: manufacturing, calibration and preliminary experimental tests on different materials, Int. J. Thermophys. 37(5) (2016) p. 47.

DOI: https://doi.org/10.1007/s10765-016-2052-2
[16] RD Rymarov s.r.o., Scheme of walls and ceilings, 2018, [Online] Available at: https://www.rdrymarov.cz/en/scheme-of-walls-and-ceilings

[17] A. Vodička, M. Maška, J. Včelák, P. Mlejnek, Sensor system for continuous moisture monitoring in wooden buildings and structures, Proc. of the World Conference on Timber Engineering, 2016, Vienna, Austria.

[18] T. Vehviläinen, H. Lindholm, H. Rintmäki, R. Pääkkönen, A. Hirvonen, O. Niemi, J. Vinha, High indoor $\mathrm{CO}_{2}$ concentrations in an office environment increases the transcutaneous $\mathrm{CO}_{2}$ level and sleepiness during cognitive work, Journal of Occupational and Environmental Hygiene 13(1) (2016) pp. 19-29.

DOI: https://doi.org/10.1080/15459624.2015.1076160

[19] J.A. Bosnic, G. Petrovic, R. Malaric, Estimation of the wall thermal properties through comparison of experimental and simulated heat flux, Proc. of the $21^{\text {st }}$ IMEKO TC4 International Symposium on Understanding the World through Electrical and Electronic Measurement, 2016, Budapest, Hungary, pp. 11-15. Online [Accessed 24 September 2020] https://www.imeko.org/publications/tc4-2016/IMEKO-TC42016-03.pdf

[20] E. Balestrieri, L. De Vito, F. Lamonaca, F. Picariello, S. Rapuano, I. Tudosa, Research Challenges in Measurement for Internet of Things systems, ACTA IMEKO 7(4) (2019) pp. 82-94. Online [Accessed 24 September 2020] DOI: http://dx.doi.org/10.21014/acta imeko.v7i4.675 